\title{
MS03-P01 | Structural Basis of Inactivation of Human Counterpart of Mouse Motor Neuron Degeneration 2 Mutant in Serine Protease Htra2
}

Wagh, Ajay (TMC-ACTREC, MUMBAI, IND)

Serine protease HtrA2 (High temperature requirement protease A2), is involved in apoptosis and protein quality control. However, one of its murine inactive mutants, (S276C aka mnd2) is associated with motor neuron degeneration 2. Similarly, this conserved mutation in human HtrA2 (hHtrA2) also renders the protease inactive, implicating pathogenicity. However, the structural determinants for its inactivation have not yet been elucidated. Here, using multidisciplinary approach, we studied the structural basis of inactivity associated with this mutation in hHtrA2. Characterization of secondary and tertiary structural properties, protein stability, oligomeric properties and enzyme activity for both wild type and mutant has been performed using biophysical and functional enzymology studies. The structural comparison at atomic resolution has been carried out using $\mathrm{X}$-ray crystallography. While enzyme kinetics showed inactivity, spectroscopic probes did not identify any significant secondary structural changes in the mutant. X-ray crystallographic analysis of the mutant protein at $2 \AA$ resolution highlighted the significance of a water molecule that plays important role in mediating intermolecular interactions for maintaining the functional ensemble of the protease. Overall, the crystallographic data, along with biophysical and enzymology studies helped decipher the structural basis of inactivity of hHtrA2S276C, which might pave way toward further investigating its correlation with aberration of normal cellular functions, hence pathogenicity. 